\title{
The intrinsic and synaptic responsiveness of a new realistic Purkinje cell model
}

\author{
Stefano Masoli ${ }^{{ }^{*}}$, Sergio Solinas ${ }^{2}$, Egidio D'Angelo ${ }^{1,2}$ \\ From Twenty Second Annual Computational Neuroscience Meeting: CNS*2013 \\ Paris, France. 13-18 July 2013
}

The latest discoveries on Purkinje cell (PC) physiology suggest that the mechanisms of PCs intrinsic excitability have to be revisited. Starting from available models [1], we have constructed a new PC model in Python-NEURON, which explicitly accounts for the Axon Initial Segment (AIS) [2-4] and a part of the axon including the first node of Ranvier (RVN). The fast $\mathrm{Na}+$ channels are located in AIS, soma with initial dendrite and RVN [4]. The K+ delayed rectifier channels are located only in the soma. The $\mathrm{Ca} 2+$ and $\mathrm{Ca} 2+-$ dependent $\mathrm{K}+$ channels, including SK2, as well as intracellular Ca2+dynamics have been updated [5]. The new model configuration now generates simple spike (SS) firing reproducing the experimental input-output curve[6]. SSs initiate in AIS and then backpropagate into the soma decaying sharply inside the dendritic tree. Activation of parallel fiber (pf) generates a short burst followed by a pause caused by Stellate cells. Following a complex spike (CS), SS activity is interrupted independently of the inhibitory synaptic input. Interestingly, the model can shift its state from silent to autorhythmic (configuring a bistable behavior) upon transient current injection or activation of CFs. The pf and granule cell ascending axon (aa) synapses have been modeled using a stochastic release mechanism activating AMPA synaptic receptors. The facilitation and depression profiles of pf and aa synapses faithfully reproduce the experimental data. This model provides a valuable tool to further investigate the Purkinje cell function in cerebellar network models.

\section{Author details}

${ }^{1}$ Department of Brain and Behavioural Science, Neurophysiology and Neurocomputation Unit, University of Pavia, Via Forlanini 6, I-27100, Pavia, Italy. ${ }^{2}$ Brain Connectivity Center, Istituto Neurologico IRCCS C. Mondino, Pavia, I-27100, Italy.

Published: 8 July 2013

\section{References}

1. De Schutter E, Bower JM: An active membrane model of the cerebellar Purkinje cell. I. Simulation of current clamps in slice. Journal of neurophysiology 1994, 71:375-400.

2. Palmer LM, Clark BA, Gr J, Roth A, Stuart GJ, Michael H: Initiation of simple and complex spikes in cerebellar Purkinje cells. Society 2010, 10:1709-1717.

3. Foust A, Popovic M, Zecevic D, Mccormick DA: Action Potentials Initiate in the Axon Initial Segment and Propagate through Axon Collaterals Reliably in Cerebellar Purkinje Neurons. Methods 2010, 30:6891-6902.

4. Zonta B, Desmazieres A, Rinaldi A, Tait S, Sherman DL, Nolan MF, Brophy PJ: $A$ critical role for Neurofascin in regulating action potential initiation through maintenance of the axon initial segment. Neuron 2011, 69:945-56.

5. Lorincz A, Nusser Z: Cell-type-dependent molecular composition of the axon initial segment. The Journal of neuroscience : the official journal of the Society for Neuroscience 2008, 28:14329-40.

6. Anwar H, Hong S, De Schutter E: Controlling Ca2+-Activated K+ Channels with Models of Ca2+ Buffering in Purkinje Cells. The Cerebellum 2010, $1-13$.

7. Khaliq ZM, Gouwens NW, Raman IM: The contribution of resurgent sodium current to high-frequency firing in Purkinje neurons: an experimental and modeling study. Journal of Neuroscience 2003, 23:4899-4912.

doi:10.1186/1471-2202-14-S1-P80

Cite this article as: Masoli et al:: The intrinsic and synaptic responsiveness of a new realistic Purkinje cell model. BMC Neuroscience 2013 14(Suppl 1):P80.

\footnotetext{
* Correspondence: stefano.masoli01@universitadipavia.it

'Department of Brain and Behavioural Science, Neurophysiology and

Neurocomputation Unit, University of Pavia, Via Forlanini 6, 1-27100, Pavia,

Italy

Full list of author information is available at the end of the article
}

(c) 2013 Masoli et al; licensee BioMed Central Ltd. This is an Open Access article distributed under the terms of the Creative Commons 